\title{
INTEGRALS AND SUMMABLE TRIGONOMETRIC SERIES
}

\author{
R. D. JAMES
}

I should like to begin by expressing my thanks to Professor Zygmund, who not only interested me in the subject of trigonometric series but also suggested the possibility of defining generalized integrals which would have applications to summable trigonometric series, and then supplied helpful hints from time to time.

1. Introduction. One of the problems in the theory of trigonometric series

$$
\frac{1}{2} a_{0}+\sum_{n=1}^{\infty}\left(a_{n} \cos n x+b_{n} \sin n x\right)=\sum_{n=0}^{\infty} a_{n}(x)
$$

is that of suitably defining a trigonometric integral with the property that, if the series (1.1) converges everywhere to a function $f(x)$, then $f(x)$ is necessarily integrable and the coefficients, $a_{n}$ and $b_{n}$, given in the usual Fourier form. It is well known that a series may converge everywhere to a function which is not Lebesgue summable nor even Denjoy integrable (completely totalisable, [3]). A simple example is that given by Fatou in which $a_{n}=0, n \geqq 0$, and $b_{n}=1 / \log (n+1)$, $n \geqq 1$.

More generally, if $\left\{b_{n}\right\}$ is any monotone decreasing sequence with limit zero such that the series $\sum b_{n} / n$ diverges, then the sum of the everywhere convergent series $\sum b_{n} \sin n x$ is not completely totalisable [5, pp. 42-44].

The problem has been solved by Denjoy $[4 ; 5]$, Verblunsky [14], Marcinkiewicz and Zygmund [10], Burkill [1; 2], and James [8]. In Verblunsky's paper and Burkill's first paper, additional hypotheses other than the convergence of (1.1) are made, and in all the papers a change in the form of the Fourier formulas is required. The solutions are described, mainly in the order in which they were published, in $\S \S 2-7$ below.

An extension of this problem is to consider series which are not necessarily convergent, but are summable $(C, k), k \geqq 1$ (Cesàro means of order $k,[6$, Chaps. V and VII $])$. Here the situation becomes more complicated since even though a series is summable to a Lebesgue

An address delivered before the Summer meeting of the Society in Laramie on August 31, 1954 by invitation of the Committee to Select Hour Speakers for Annual and Summer Meetings; received by the editors September 2, 1954. 
summable function, the coefficients may not be given by the usual formulas. In particular, there are series such as $\sum n \sin n x$ which is summable $(C, 2)$ to zero for all $x$, whereas the coefficients are not zero. It is difficult to conceive of a process of integration which would integrate an identically zero function and yield anything but zero. Wolf [15] has solved the problem for series summable $(C, k)$ to a Denjoy integrable function and his results show that there may be a reducible set of exceptional values of $x$ and that these exceptional values enter into the formulas for the coefficients. Since Wolf was not concerned with the question of a trigonometric integral, his work will not be discussed in this paper.

There is another difference between convergent and summable trigonometric series which makes the latter more difficult to deal with. The formal product ([6, Chap. X], [16], [17, Chap. XI]) of the series (1.1) and, for example, $\sin m x$ is the trigonometric series obtained by muliplying (1.1) by $\sin m x$ and replacing the products $\cos n x \sin m x, \sin n x \sin m x$ by sums of cosines and sines. It is easy to see that, if the series (1.1) converges to $f(x)$, then the formal product of (1.1) and $\sin m x$ converges to $f(x) \sin m x$. This statement is not true in general for summable trigonometric series. A simple example due to Rajchman $[16$, p. 78$]$ is the series $\sum \sin n x$, which is summable $(C, 1)$ to $2^{-1} \cot (x / 2)$ for $x \neq 0$, and to zero for $x=0$. The formal product of this series and $\sin x$ is, however, $(1+\cos x) / 2$, which is equal to 1 when $x=0$. Thus it is possible that a process of integration designed for series summable $(C, 1)$ would integrate $f(x)$ but not $f(x) \sin x$, and the usual Fourier formulas could not be valid.

The problem of suitably defining a trigonometric integral which may be applied to trigonometric series summable $(C, k)$ can be solved if an additional condition involving the series

$$
\sum_{n=1}^{\infty}\left(a_{n} \sin n x-b_{n} \cos n x\right)=-\sum_{n=1}^{\infty} b_{n}(x),
$$

conjugate to (1.1), is imposed. The condition is that the series $\sum\left\{b_{n}(x)-b_{n+1}(x)\right\}$ be summable $(C, k)$ to $b_{1}(x)$ or, equivalently, that $b_{n}(x) \rightarrow 0,(C, k)$. In the case of convergent series $(k=0)$, this condition is automatically satisfied. With this extra condition, it can be shown that the generalized $\mathrm{P}^{k+2}$-integral [9] integrates trigonometric series summable $(C, k)$ and that the coefficients are given by a suitable modification of the Fourier formulas. A description of the method, which has not yet been published, is given in $\$ 8$ below.

2. Totalisation of generalized second derivatives. The process of 
totalisation introduced by Denjoy goes back to 1912 and the extension to the problem of calculating a second primitive (totalisation symétrique à deux degrés) was outlined by him in a series of notes in 1921 [4]. The publication of a detailed account was begun in 1941 and, delayed by the war, completed in 1949 [5].

The application to trigonometric series is based on a fundamental result due to Riemann. Let $\theta_{2}(x, h)=\theta_{2}(F ; x, h)$ be defined by

$$
\frac{h^{2}}{2 !} \theta_{2}(x, h)=\frac{1}{2}\{F(x+h)+F(x-h)\}-F(x) .
$$

If the limit of $\theta_{2}(x, h)$ as $h \rightarrow 0$ exists it is called the generalized (symmetric) second derivative of $F(x)$ and written $D^{2} F(x)$. Riemann proved that, if the series (1.1) converges for all $x$ to a function $f(x)$, then the series

$$
\frac{1}{2} a_{0} \frac{x^{2}}{2 !}-\sum_{n=1}^{\infty} \frac{a_{n}(x)}{n^{2}}
$$

(obtained by integrating (1.1) formally term-by-term twice) converges uniformly to a continuous function $F(x)$ such that $D^{2} F(x)$ $=f(x)$. This suggests the problem of defining a process of integration, which, starting with the generalized second derivative of a continuous function, yields the function itself.

Denjoy extended the problem to include the case where the generalized second derivative may not exist for all $x$ and considered generalized second derivates. The lim sup and lim inf of $\theta_{2}(x, h)$ as $h \rightarrow 0$ are called, respectively, the upper and lower (extreme) generalized second derivates and denoted by $\Delta^{2} F(x), \delta^{2} F(x)$. It may happen that $\theta_{2}\left(x, h_{n}\right)$ tends to other limits for suitably chosen sequences $\left\{h_{n}\right\}$ tending to zero. Such limits are called median generalized second derivates. The problem he set is that of finding a process of totalisation for a function $f(x)$, knowing only that it is one of the generalized second derivates (extreme or median) of a continuous function with finite extreme generalized second derivates at each point of an interval $(a, b)$. The process should determine the second primitive $F(x)$ up to an arbitrary linear function, or, equivalently, determine the second variation of $F(x)$ defined by

$$
V(F ; \alpha, \beta, \gamma)=(\gamma-\beta) F(\alpha)+(\alpha-\gamma) F(\beta)+(\beta-\alpha) F(\gamma)
$$

for any three points, $\alpha, \beta, \gamma$, of $(a, b)$.

The starting point of the attack on the problem is the following result $[5, \S 52 \mathrm{~b}, 2$, pp. 219-220]: 
Suppose that the maximum of $\left|\theta_{2}(x, h)\right|$ for all $h$ is a finite number $A(x)$ for $x$ belonging to a perfect set $P$ contained in $(a, b)$. Then, either $A(x)$ is bounded by a number $A(P)$ independent of $x \in P$, or the set $E$ of points of $P$ in the neighborhood of which $A(x)$ is not bounded is nondense in $P$.

Since $\Delta^{2} F(x)$ and $\delta^{2} F(x)$ are supposed finite in $(a, b)$ the maximum of $\theta_{2}(x, h)$ is also finite, and the hypothesis above is satisfied with $P=(a, b)$. In every closed sub-interval of $(a, b)$ having no point in common with $E, A(x)$ is bounded and hence $f(x)$, being one of the generalized second derivatives, is also bounded in this sub-interval. It follows that the set $S_{1}=S_{1}((a, b), f)$ of points of $(a, b)$ in the neighborhood of which $f(x)$ is not Lebesgue summable is nondense in $(a, b)$. Thus if $\alpha, \beta, \gamma$ are any three points in the same interval contiguous to $S_{1}$, the second variation of $F(x)$ is given by

$$
\begin{aligned}
V(F ; \alpha, \beta, \gamma)= & \int_{\beta}^{\gamma}(\beta \gamma+\alpha x) f(x) d x \\
& +\int_{\gamma}^{\alpha}(\gamma \alpha+\beta x) f(x) d x+\int_{\alpha}^{\beta}(\alpha \beta+\gamma x) f(x) d x,
\end{aligned}
$$

where the integrals are taken in the Lebesgue sense.

Denjoy defines what he calls problem (U) relative to a set $E$ in $(a, b)$ as that of calculating $V(F ; \alpha, \beta, \gamma)$ for any three points $\alpha, \beta, \gamma$, of $E$. Problem (U) contiguous to a set $E$ is said to be solved if problem (U) relative to each interval contiguous or semi-contiguous to $E$ has been solved. Using this terminology, what has just been shown is that problem (U) is solved (by Lebesgue integrals) contiguous to the set $S_{\mathbf{1}}$. (This Lebesgue integration is named operation 1 by Denjoy [5, p. 277].)

Two further operations (2 and 3) lead from the solution of problem (U) contiguous to $S_{1}$ to the solution of problem (U) contiguous to $S_{1}^{\prime}$, the first derivative of $S_{1}$. The two operations involve the taking of limits. In general, if $\mu$ is an ordinal number of the first kind and problem (U) contiguous to $S_{1}^{(\mu-1)}$, the derivative of order $\mu-1$ of $S_{1}$, has been solved, a finite number of applications of operation 3 leads to the solution contiguous to $S_{1}^{(\mu)}$. If $\mu$ is of the second kind and problem (U) contiguous to $S_{1}^{(\nu)}$ has been solved for every $\nu<\mu$, then it is also solved contiguous to $S_{1}^{(\mu)}$. Eventually, after at most a countable number of applications of operations 2 and 3, the solution is found contiguous to $P_{1}$, the maximal perfect set contained in $S_{1}$. The set $P_{1}$ is called by Denjoy [5, p. 91], the perfect nucleus of $S_{1}$. The conclusion now is that problem (U) contiguous to $P_{1}$ is solved. 
Unlike the problem of simple (or complete) totalisation $[5, \S 76 \mathrm{~b}$, 2, p. 337], it is not known a priori that $P_{1}$ contains a closed set $S_{2}$, nondense in $P_{1}$, such that problem (U) contiguous to $S_{2}$ may be solved. Hence another method must be found.

It may happen that $P_{1}$ is one of three special types of perfect sets $\left[5, \S 39\right.$, pp. 121-126], and that $A(x)$ is bounded on $P_{1}$. In this case, Denjoy shows, using possibly six more operations $(4,5,6,7,8,9)$, that problem (U) relative to $(a, b)$ can be solved. This involves intricate analysis and a great deal of detailed calculation. The operations themselves consist of a Lebesgue integration of $f(x)$ and $x f(x)$ over a perfect discontinuous set where $f(x)$ is summable, completed by the summation of an absolutely convergent series and the taking of various limits. There is a recapitulation of the role played by each of the nine operations in $[5, \S 73 \mathrm{e}, \mathrm{pp} .317-318]$.

If $P_{1}$ is not one of the three special types, or if $A(x)$ is not bounded on $P_{1}$, it becomes necessary to construct a closed set $S_{2} \subset P_{1}$ which is nondense in $P_{1}$ and such that problem (U) contiguous to $S_{2}$ may be solved $[5, \S 74$, pp. 318-325]. Then the above argument can be repeated with $S_{2}$ in place of $S_{1}$.

In this way a sequence $\left\{S_{\mu}, P_{\mu}\right\}$ of pairs of sets is constructed with $S_{\mu}$ closed and $P_{\mu}$ the perfect nucleus of $S_{\mu}$. If at any stage $P_{\mu}$ is one of the three special types and $A(x)$ is bounded on $P_{\mu}$, problem (U) relative to $(a, b)$ is solved. Otherwise, the sequence $S_{\mu}$ of closed sets, each containing the next (equality excluded) must terminate so that all the sets $S_{\mu}$ beyond a certain $S_{\nu}$ are empty. Then problem (U) relative to $(a, b)$ is again solved.

The calculation of the coefficients of a trigonometric series which converges for all $x$ to a finite function $f(x)$ is now an easy matter. The second variation of the function $F(x)$ obtained by totalisation from $f(x)$ is the second variation of the series (2.2): This, for the particular points $-2 \pi, 0,2 \pi$ gives

$$
4 \pi^{3} a_{0}=V(F,-2 \pi, 0,2 \pi) .
$$

The formal products of (1.1) and $\cos m x$ and of (1.1) and $\sin m x$ converge for all $x$ to $f(x) \cos m x$ and $f(x) \sin m x$, respectively. The constant terms in the new series are $a_{m} / 2$ and $b_{m} / 2$, respectively. Hence, if $G_{m}(x)$ and $H_{m}(x)$ denote the functions obtained by totalisation from $f(x) \cos m x$ and $f(x) \sin m x$, respectively, then

$$
\begin{aligned}
& 4 \pi^{3} a_{m}=V\left(G_{m},-2 \pi, 0,2 \pi\right), \\
& 4 \pi^{3} b_{m}=V\left(H_{m},-2 \pi, 0,2 \pi\right) .
\end{aligned}
$$

Thus the problem for a convergent trigonometric series is com- 
pletely solved. The solution may seem unnecessarily complicated and Denjoy himself remarks [5, p. 325] "Certains de mes lecteurs me feront peut-être grief de la minutie des calculs développés dans les cinquantes pages de ce Chapitre (X)." It must be pointed out, however, that he gives examples [5, §114, pp. 585-593], of trigonometric series which converge for all $x$ and for which the last five operations are required. In addition he shows that it is impossible that the sequence $\left\{S_{\mu}, P_{\mu}\right\}$ of pairs of sets be such that there is a unique $\mu_{0}$ beyond which all the sets $S_{\mu}$ are empty for every convergent trigonometric series.

3. Approximate totalisation. Instead of using the series (2.2) Verblunsky [14] considered the series found by a single formal integration term-by-term of (1.1). In addition he restricted his attention to the complex power series

$$
\begin{aligned}
& \sum_{n=1}^{\infty} c_{n} e^{i n x}, \\
& \sum_{n=1}^{\infty} c_{n} e^{i n x} / i n,
\end{aligned}
$$

where $c_{n}=a_{n}-i b_{n}$. It is still true, however, that a process more powerful than totalisation is needed. There are series of the form (3.1) convergent for all $x$, such that (3.2) is not uniformly convergent [11], whereas the indefinite Denjoy integral is necessarily continuous.

Denjoy defines a class of functions which he calls résoluble (cf. [13, Chapter VII, particularly §9]). A function of this class has an approximate derivative almost everywhere which is (simply) totalisable. Verblunsky widens the class to approximately resoluble functions which still have an approximate derivative almost everywhere. An approximately resoluble function in $(a, b)$ is one that is a differential coefficient and such that every perfect set in $(a, b)$ contains a portion on which the function is resoluble.

One of the conditions imposed on a function $f(x)$ in the process of totalisation is that the set of points of $(a, b)$ in the neighbourhood of which $f(x)$ is not Lebesgue summable is nondense in $(a, b)$. Verblunsky replaces "not Lebesgue summable" by "not totalisable." It is then possible to define approximate totalisation, a process which integrates the approximate derivative of any approximately resoluble function.

Verblunsky's result is as follows [14, Theorem V]:

If the partial sums of the series (3.1) are bounded for all $x$ except possibly those belonging to a countable set, and if the series (3.2) con- 
verges for all $x$ to $G(x)-i H(x)$, then the approximate derivatives $G_{a}(x)$, $H_{a}(x)$ exist almost everywhere and

$$
\begin{aligned}
& \pi a_{m}=\int_{-\pi}^{\pi} G_{a}(x) \cos m x d x=-\int_{-\pi}^{\pi} H_{a}(x) \sin m x d x, \\
& \pi b_{m}=\int_{-\pi}^{\pi} G_{a}(x) \sin m x d x=\int_{-\pi}^{\pi} H_{a}(x) \cos m x d x
\end{aligned}
$$

where the integrals are taken in the approximately totalisable sense. If, in addition, the series (3.1) is Abel (or Poisson) summable to $g(x)-i h(x)$ almost everywhere, then $G_{a}(x)=g(x), H_{a}(x)=h(x)$ almost everywhere and $a_{m}, b_{m}$ are given by the usual Fourier formulas.

4. The integral of Marcinkiewicz and Zygmund. One method of defining a process of integration is through the use of majorants and minorants. If $f(x)$ is defined in an interval $(a, b)$, a majorant of $f(x)$ in the ordinary Perron sense is a function $M(x)$ satisfying the conditions

$$
\begin{gathered}
M(x) \text { is continuous in }(a, b), \\
M(a)=0,
\end{gathered}
$$

(4.3) $D_{*} M(x) \geqq f(x)$, almost everywhere in $(a, b)$,

(4.4) $D_{*} M(x) \neq-\infty$, except possibly at a countable set in $(a, b)$.

In this definition $D_{*} M(x)$ denotes the (ordinary) lower derivate of $M(x)$. A minorant $m(x)$ is similarly defined with $D^{*} m(x) \leqq f(x)$ and $D^{*} m(x) \neq \infty$ replacing (4.3) and (4.4), respectively. Let $J(b)$ denote the inf of the numbers $M(b)$ for all majorants and $j(b)$ the sup of the numbers $m(b)$ for all minorants. If $J(b)=j(b)$, the common value is the Perron integral of $f(x)$ over $(a, b)$.

Since the Perron integral and totalisation (complete) are equivalent, it is clear that some extension of the classes of majorants and minorants is needed for applications to trigonometric series. Marcinkiewicz and Zygmund [10] altered condition (4.1) to the requirement that $M(x)$ be continuous in mean, that is, $M(x)$ is Perron integrable and

$$
\lim _{h \rightarrow 0} \frac{1}{h} \int_{0}^{h} M(x+t) d t=M(x),
$$

for all $x \in P$, where $P \subset(a, b), a \in P, b \in P$, and $(a, b)-P$ is of measure zero. They also replaced the ordinary lower derivates in (4.3) and (4.4) by the lower Borel symmetric derivate, $B D_{*} M(x)$ defined as the lim inf as $h \rightarrow 0$ of 


$$
\frac{1}{h} \int_{0}^{h} \frac{M(x+t)-M(x-t)}{2 t} d t .
$$

Similar changes were made in the definition of a minorant. If $J(b)$ $=j(b)$, the common value $F(b)$ is the MZ-integral of $f(x)$ with respect to $P$ over $(a, b)$ and may be written

$$
F(b)=\left(a \int_{P} b\right) f(x) d x .
$$

One of the properties of the integral is that, for $\alpha \in P, \beta \in P$,

$$
F(\beta)-F(\alpha)=\left(\alpha \int_{P}^{\beta}\right) f(x) d x .
$$

The integral is not defined for all $\alpha, \beta$, but only for almost all $\alpha, \beta$, in $(a, b)$. This, surprisingly enough, is an advantage, rather than a disadvantage for applications to trigonometric series. The reason is as follows. If the series (1.1) converges to $f(x)$ for all $x$, the series

$$
\frac{1}{2} a_{0} x-\sum_{n=1}^{\infty} \frac{b_{n}(x)}{n}
$$

(obtained from (1.1) by integrating formally term-by-term) converges almost everywhere to a function $G(x)$ which is continuous in mean. If $F(x)$ denotes the sum of the series (2.2), the fundamental result of Riemann (\$2) shows that $D^{2} F(x)=f(x)$. But, it is not difficult to show that whenever $D^{2} F(x)$ exists, so do $B D_{*} G(x)$ and $B D^{*} G(x)$ and that each is equal to $D^{2} F(x)=f(x)$. Hence, if $P$ denotes the set of points where $G(x)$ exists and is continuous in mean, and if $\alpha \in P$, then $G(x)-G(\alpha)$ is both a (Borel) majorant and a minorant of $f(x)$. It follows that

$$
G(\alpha+2 \pi)-G(\alpha)=\left(\alpha \int_{P} \alpha+2 \pi\right) f(x) d x .
$$

But, from (4.7), the left side of (4.8) is equal to $\pi a_{0}$, which gives the first of the Fourier formulas. The others are found by the process of formal multiplication explained at the end of $\$ 2$.

5. The Cesàro-Perron integral. Burkill defined a process of integration which is similar to that of Marcinkiewicz and Zygmund. $\mathrm{He}$ altered (4.1) in the same way although he used the term Cesàro- or $\mathrm{C}$-continuous instead of continuous in mean. He replaced the ordinary lower derivate in (4.3) and (4.4) by the lower Cesàroderivate, $C D_{*} M(x)$, defined as the lim inf as $h \rightarrow 0$ of 


$$
\frac{2}{h^{2}} \int_{0}^{h}\{M(x+t)-M(x)\} d t .
$$

Similar changes were made in defining a minorant. If $J(b)=j(b)$, the common value $F(b)$ is the Cesàro-Perron or CP-integral of $f(x)$ over $(a, b)$, and may be written

$$
F(b)=(\mathrm{CP}) \int_{a}^{b} f(x) d x .
$$

This integral also applies to the problem considered by Verblunsky (\$3). It turns out [1] that the first two conditions imposed by Verblunsky imply (in the notation of $\S 3$ ) that $G(x)$ and $H(x)$ have finite upper and lower Cesàro derivates in $(a, b)$ with the possible exception of a countable set. From this it follows that the upper and lower Cesàro derivates are equal almost everywhere, and, denoting the common values by $C D G(x), C D H(x)$, that each of $C D G(X)$, $C D H(x)$ is CP-integrable over $(a, b)$. Moreover, the coefficients $a_{m}, b_{m}$ are given by (3.3) and (3.4), respectively, with $G_{a}(x), H_{a}(x)$ replaced by $C D G(x), C D H(x)$, respectively, and the integrals by CP-integrals.

Burkill [1] also shows that the third condition of Verblunsky that the series (3.1) is Abel summable almost everywhere is redundant, being implied by the other two.

6. The Perron second integral. James and Gage [7] defined a Perron second (or $\mathrm{P}^{2}$-integral) which, like Denjoy's totalisation of a generalized second derivate, starts with a function $f(x)$ and goes directly to a second primitive $F(x)$ such that $D^{2} F(x)=f(x)$ almost everywhere.

They made no change in condition (4.1) but required $M(x)$ to vanish at both ends of the interval $(a, b)$. They also replaced the ordinary lower derivate in (4.3) and (4.4) by the lower generalized second derivate $\delta^{2} M(x)$. In addition the condition of smoothness,

$$
h \theta_{2}(x, h) \rightarrow 0 \text { as } h \rightarrow 0,
$$

was required to hold for all $x$ in the countable set of exceptional points of (4.4). If $c$ is any point of $(a, b)$, let $-J(c)$ denote the inf of the numbers $-M(c)$ for all majorants and $-j(c)$ the sup of the numbers $-m(c)$ for all minorants. If $-J(c)=-j(c)$, the common value $-F(c)$ is the $\mathrm{P}^{2}$-integral of $f(x)$ over $(a, b ; c)$ and may be written

$$
-F(c)=\int_{(a, b)}^{c} f(x) d_{2} x .
$$


The reason for using negative signs is that $M(x)-m(x)$ is convex in $(a, b)$ and this makes $-M(x) \geqq-m(x)$.

In a second paper, James [8] showed that the $\mathrm{P}^{2}$-integral provides a solution of Denjoy's problem (U) relative to the interval $(a, b)$. In fact, if $f(x)$ is $\mathrm{P}^{2}$-integrable over $(a, b ; c)$ it is also integrable over $(\alpha, \beta ; \gamma)$, where $a \leqq \alpha<\gamma<\beta \leqq b$, and, in addition,

$$
(\beta-\alpha) \int_{(\alpha, \beta)}^{\gamma} f(x) d_{2} x=V(F ; \alpha, \beta, \gamma) .
$$

Hence, if $f(x)$ and $F(x)$ are the sums of the series (1.1) and (2.2), respectively, Denjoy's results (2.5), (2.6) show that

$$
\pi^{2} a_{m}=\int_{(-2 \pi, 2 \pi)}^{0} f(x) \cos m x d_{2} x, \quad \pi^{2} b_{m}=\int_{(-2 \pi, 2 \pi)}^{0} f(x) \sin m x d_{2} x .
$$

Thus the $\mathrm{P}^{2}$-integral also provides a complete solution to the problem for convergent trigonometric series.

It follows from a general result of Denjoy $[5, \S 66]$ that a function with a finite generalized second derivative almost everywhere also has a derivative $F^{\prime}(x)$ almost everywhere. In the case of trigonometric series, this derivative is equal to sum of the series (4.7) wherever it converges. Hence another expression for the Fourier coefficient $a_{0}$ is

$$
\pi a_{0}=F^{\prime}(\alpha+2 \pi)-F^{\prime}(\alpha)
$$

where $\alpha \in P$, the set of points at which the series (4.7) converges. There are similar expressions for the other coefficients using the derivatives of the functions $G_{m}(x), H_{m}(x)$ defined in $\$ 2$.

7. The symmetric Cesàro-Perron integral. Burkill [2] noted that the $\mathrm{CP}$ indefinite integral, being defined for all $x$, was not suitable for applications to trigonometric series because the series (4.7) need converge only almost everywhere even when the series (1.1) converges everywhere. He therefore modified his definition of the CP-integral in order to have one of the same type as the MZ-integral ( $\$ 4$ ).

He altered condition (4.1) to the condition (4.5) and replaced the lower Cesàro-Perron derivate by the lower symmetric Cesàro-Perron derivate, $S C D_{*} M(x)$, defined as the lim inf as $h \rightarrow 0$ of

$$
\frac{1}{h^{2}} \int_{0}^{h}\{M(x+t)-M(x-t)\} d t .
$$

He also required $M(x)$ to satisfy 


$$
\frac{1}{h} \int_{0}^{h}\{M(x+t)-M(x-t)\} d t \rightarrow 0 \quad \text { as } \quad h \rightarrow 0
$$

in addition to (4.5) at the countable set of points where $S C D_{*} M(x)$ $=-\infty$.

If $T(x)$ is the integral of $M(x)$, then $S C D_{*} M(x)=\delta^{2} T(x)$ and condition (7.2) is simply the requirement (6.1) of smoothness. It can also be shown [10, Lemma 40] that

$$
\begin{aligned}
\delta^{2} T(x) & =S C D_{*} M(x) \leqq B D_{*} M(x), \\
B D^{*} m(x) & \geqq S C D^{*} m(x)=\Delta^{2} t(x) .
\end{aligned}
$$

Hence any function which is SCP-integrable is necessarily MZ- and $\mathrm{P}^{2}$-integrable, and the formulas for the coefficients are of the same form.

As far as the integration of convergent trigonometric series is concerned, the three types of integrals are equivalent. Whether or not the $\mathrm{MZ}$ - or $\mathrm{P}^{2}$-integral is actually more general than the SCPintegral is not yet known.

8. The $\mathrm{P}^{n}$-integral. James [9] extended the $\mathrm{P}^{2}$-integral by replacing generalized second derivates by generalized symmetric derivates of higher order, but required that the inequalities analogous to (4.3) and (4.4) hold without exception. In addition a condition similar to the smoothness condition (6.1) was imposed at all points of $(a, b)$ with the possible exception of a countable set. Only the case $n=4$ will be considered here since it fully illustrates the difference between the general case and the case $n=2$.

If $M(x)$ is continuous and $D^{2} M(x)$ is finite in $(a, b)$, let $\theta_{4}(x, h)$ $=\theta_{4}(M ; x, h)$ be defined by

$$
\frac{h^{4}}{4 !} \theta_{4}(x, h)=\frac{1}{2}\{M(x+h)+M(x-h)\}-M(x)-\frac{h^{2}}{2 !} D^{2} M(x) .
$$

The lim sup and $\lim$ inf as $h \rightarrow 0$ of $\theta_{4}(x, h)$ are the upper and lower generalized symmetric derivates of order $4, \Delta^{4} M(x), \delta^{4} M(x)$, respectively. If the two are equal, their common value is the generalized symmetric derivative of order $4, D^{4} M(x)$.

The main difference between the general case and the case $n=2$ is that the analogue of the theorem of Schwarz does not hold. This theorem states that, if $D^{2} M(x)=0$ throughout an interval $(a, b)$, then $M(x)$ is a linear function. It is not true, for example, that if $D^{4} M(x)=0$ in $(a, b)$, then $M(x)$ is a cubic function. A simple example given by M. Riesz [12] is the function defined by 


$$
M(x)=\left\{\begin{aligned}
x^{2}, & x \geqq 0 \\
-x^{2}, & x \leqq 0
\end{aligned}\right.
$$

It is clear that $M(x)$ is continuous, that

$$
D^{2} M(x)=\left\{\begin{aligned}
2, & x>0, \\
0, & x=0, \\
-2, & x<0,
\end{aligned}\right.
$$

and that $D^{4} M(x)$ is identically zero. Obviously $M(x)$ is not a cubic function. The interesting part of the example is that $D^{2} M(x)$ has an ordinary discontinuity at $x=0$. If such a possibility is ruled out, the analogue of the theorem of Schwarz is true. It follows from [9, Theorem 4.2] (with $m=2$ ) that if $M(x)$ is continuous, $D^{2} M(x)$ is finite and has no ordinary discontinuities, and $D^{4} M(x)=0$ in $(a, b)$, then $M(x)$ is a cubic function. In defining a majorant or minorant using generalized derivates of order 4 , the condition that the generalized second derivative have no ordinary discontinuities must be imposed.

The definition of a majorant (in the $\mathrm{P}^{4}$ sense) follows the pattern of $\$ \S 4$ and 6 . The function $M(x)$ is required to be continuous and to vanish at four points $a_{i}, i=1, \cdots, 4$, such that $a=a_{1}<a_{2}<a_{3}<a_{4}$ $=b$. Conditions (4.3) and (4.4) hold without exception with $D_{*} M(x)$ replaced by $\delta^{4} M(x)$, and the smoothness condition (6.1) becomes $h \theta_{4}(x, h) \rightarrow 0$ as $h \rightarrow 0$ for all $x$ in $(a, b)$ with the possible exception of a countable set. In addition, $D^{2} M(x)$ has no ordinary discontinuities in $(a, b)$. A minorant is defined in a similar way.

With each majorant and minorant there is associated the function defined by

$$
\begin{aligned}
M^{*}(x)=(-1)^{r} M(x), \quad m^{*}(x)=(-1)^{r} m(x), \text { for } \quad a_{r} \leqq x \leqq & a_{r+1}, \\
r & =1,2,3 .
\end{aligned}
$$

The point of the last definition is that $M(x)-m(x)$ is 4-convex $[9, \S 2]$ and that then $M^{*}(x) \geqq m^{*}(x)$. If $c$ is any point of $(a, b)$, let $J^{*}(c)$ denote the inf of the numbers $M^{*}(c)$ for all majorants and $j^{*}(c)$ the sup of the numbers $m^{*}(c)$ for all minorants. If $J^{*}(c)=j^{*}(c)$, the function $f(x)$ is $\mathrm{P}^{4}$-integrable over $\left(a_{i} ; c\right)=\left(a_{1}, a_{2}, a_{3}, a_{4} ; c\right)$. In this case the function defined by $(-1)^{\circ} F(c)=J^{*}(c)=j^{*}(c)$ when $a_{s}<c$ $<a_{8+1}, s=1,2,3$, is the $\mathrm{P}^{4}$-integral and may be written

$$
(-1)^{8} F(c)=\int_{\left(a_{i}\right)}^{c} f(x) d_{4} x .
$$


Since $J^{*}\left(a_{i}\right)=j^{*}\left(a_{i}\right)=0$, the integral is zero if $c=a_{i}, i=1, \cdots, 4$.

The $\mathrm{P}^{4}$-integral integrates the generalized derivative of order 4 of any continuous function $G(x)$ provided that $D^{2} G(x)$ has no ordinary discontinuities. In fact,

$$
(-1)^{\circ} \int_{\left(a_{i}\right)}^{c} D^{4} G(x) d_{4} x=G(c)-\sum_{i=1}^{4} \lambda\left(c ; a_{i}\right) G\left(a_{i}\right),
$$

where $\lambda\left(x ; a_{i}\right)=\prod_{j \neq i}\left(c-a_{j}\right) /\left(a_{i}-a_{j}\right)$ is a polynomial of the third degree in $c$.

The application of the $\mathrm{P}^{4}$-integral to summable trigonometric series is based on an extension $([15$, Theorem $B]$ and $[17, \$ 10.42])$ of Riemann's result for the generalized second derivative. For $n=4$ the extension is as follows:

If the trigonometric series $(1.1)$ is summable $(C, 2)$ for all $x$ to a function $g(x)$, then the series

$$
\frac{1}{2} a_{0} \frac{x^{4}}{4 !}+\sum_{n=1}^{\infty} \frac{a_{n}(x)}{n^{4}}
$$

(obtained by integrating (1.1) formally term-by-term four times) converges uniformly to a function $G(x)$ such that $D^{4} G(x)=g(x)$.

It also follows, of course, that $D^{2} G(x)$ is the sum of the convergent series (2.2), but it is not true, in general, that $D^{2} G(x)$ has no ordinary discontinuities. For example, the series $\sum n \sin n x$ is summable $(C, 2)$ to zero for all $x$, but the series $-\sum(\sin n x) / n$ converges to $-(\pi+x) / 2$ if $-2 \pi<x<0$, to zero if $x=0$, and to $-(\pi-x) / 2$ if $0<x<2 \pi$. Thus there is an ordinary discontinuity at $x=0$.

The difficulty is overcome by imposing a condition on the coefficients of the series (4.7) obtained by integrating (1.1) formally termby-term. The condition is that $b_{n}(x) / n \rightarrow 0(C, 1)$, which is equivalent to requiring that the series $\sum\left\{b_{n}(x) / n-b_{n+1}(x) /(n+1)\right\}$ be summable $(C, 1)$ to $b_{1}(x)$. It then follows by a slight modification of Zygmund's argument $[17, \S 10.42]$ that $G^{\prime}(x)$ exists and that

$$
D^{2} G(x)=\lim _{h \rightarrow 0} \frac{G(x+h)-G(x)-h G^{\prime}(x)}{h^{2} / 2 !} .
$$

Hence $D^{2} G(x)$ is equal to $G_{(2)}(x)$, the second differential coefficient of $G(x)$, which by [9, Lemma 8.1] cannot have an ordinary discontinuity. In the example above, $b_{n}(x) / n=\cos n x$, which for $x=0$, the point of discontinuity of $D^{2} G(x)$, does not tend to zero $(C, 1)$.

The condition $b_{n}(x) / n \rightarrow 0(C, 1)$ is not strong enough to deal with formal multiplication of summable trigonometric series. However, if 
$b_{n}(x) \rightarrow 0(C, 2)$, then the formal products of (1.1) and $\cos m x$ and $\sin m x$ are summable $(C, 2)$ to $g(x) \cos m x$ and $g(x) \sin m x$ whenever (1.1) is summable $(C, 2)$ to $g(x)$. Zygmund proves this for Abel summability and indicates in a footnote $[16$, p. 79$]$ that analogous results hold for Cesàro summability.

Since $b_{n}(x) \rightarrow 0(C, 2)$ implies that $b_{n}(x) / n \rightarrow 0(C, 1)$, the stronger condition is used in the final result which may be summed up as follows:

If the trigonometric series $(1.1)$ is summable $(C, 2)$ to a function $g(x)$ and if $b_{n}(x) \rightarrow 0(C, 2)$ for all $x$, then $g(x), g(x) \cos m x, g(x) \sin m x$ are $\mathrm{P}^{4}$-integrable. It also follows from (8.1) that the coefficients of (1.1) are given by

$$
\begin{aligned}
& \frac{4 \pi^{4}}{3} a_{m}=\int_{\left(a_{i}\right)}^{0} g(x) \cos m x d_{4} x, \\
& \frac{4 \pi^{4}}{3} b_{m}=\int_{\left(a_{i}\right)}^{0} g(x) \sin m x d_{4} x,
\end{aligned}
$$

where $\left(a_{i}\right)=(-4 \pi,-2 \pi, 2 \pi, 4 \pi)$.

The general result takes the same form with summability $(C, 2)$ replaced by summability $(C, k), k \geqq 1$, and the $\mathrm{P}^{4}$-integral by the $\mathrm{P}^{k+2}$-integral. The numerical coefficients of $a_{m}$ and $b_{m}$ in (8.3) and (8.4) and the set $\left(a_{i}\right)$ have different forms according as $k$ is even or odd. If $k=2 r-2$, the numerical coefficient is $2^{2 r-1} \pi^{2 r}(r !)^{2} /(2 r) !$ and

$$
\left(a_{i}\right)=(-2 r \pi, \cdots,-2 \pi, 2 \pi, \cdots, 2 r \pi) .
$$

If $k=2 r-1$, the numerical coefficient is $2^{2 r} \pi^{2 r+1} r !(r+1) ! /(2 r+1) !$ and

$$
\left(a_{i}\right)=(-(2 r+1) \pi, \cdots,-3 \pi,-\pi, 3 \pi, \cdots,(2 r+1) \pi)
$$

and the 0 in the integral becomes $\pi$.

Thus, if a trigonometric series is summable $(C, k)$ and the coefficients of the conjugate series tend to zero $(C, k)$ for all $x$, the $\mathrm{P}^{k+2}$ integral provides a complete solution to the problem of expressing the coefficients in terms of integrals analogous to the usual Fourier formulas.

\section{REFERENCES}

1. J. C. Burkill, The expression of trigonometrical series in Fourier form, J. London Math. Soc. vol. 11 (1936) pp. 43-48.

2. - Integrals and trigonometric series, Proc. London Math. Soc. (3) vol. 1 (1951) pp. 46-57.

3. A. Denjoy, La totalisation des nombres dérivés non sommables, Ann. École Norm. (3) vol. 33 (1916) pp. 127-222; vol. 34 (1917) pp. 181-236. 
4. - Calcul des coefficients d'une série trigonométrique partout convergente, C. R. Acad. Sci. Paris vol. 172 (1921) pp. 653-655, 833-835, 903-906, 1218-1221; vol. 173 (1921) pp. 127-129.

5. - Leçons sur le calcul des coefficients d'une série trigonometrique, Paris, 1941 and 1949.

6. G. H. Hardy, Divergent series, Oxford, 1949.

7. R. D. James and Walter H. Gage, $A$ generalized integral, Trans. Roy. Soc. Canada, Sect. III vol. 40 (1946) pp. 25-35.

8. R. D. James, $A$ generalized integral II, Canadian Journal of Mathematics vol. 2 (1950) pp. 297-306.

9. - Generalized nth primitives, Trans. Amer. Math. Soc. vol. 76 (1954) pp. $149-176$.

10. J. Marcinkiewicz and A. Zygmund, On the differentiability of functions and summability of trigonometrical series, Fund. Math. vol. 26 (1936) pp. 1-43.

11. L. Neder, Zur Theorie der trigonometrischen Reihen, Math. Ann. vol. 84 (1921) pp. 117-136.

12. M. Riesz, Über summierbare trigonometrische Reihen, Math. Ann. vol. 71 (1912) pp. 54-75.

13. S. Saks, Theory of the integral, Warsaw, 1937.

14. S. Verblunsky, On the theory of trigonometric series VII, Fund. Math. vol. 23 (1934) pp. 193-235.

15. F. Wolf, Summable trigonometric series: an extension of uniqueness theorems, Proc. London Math. Soc. (2) vol. 45 (1939) pp. 328-356.

16. A. Zygmund, Sur la thêrie riemannienne des séries trigonométriques, Math. Zeit. vol. 24 (1926) pp. 47-104.

17. —, Trigonometrical series, Warsaw, 1935.

University of British Columbia 